\title{
Mechanisms of aortic valve incompetence: Finite-element modeling of Marfan syndrome
}

\author{
K. Jane Grande-Allen, $\mathrm{PhD}^{\mathrm{a}}$ \\ Richard P. Cochran, $\mathrm{MD}^{\mathrm{b}}$ \\ Per G. Reinhall, PhDc \\ Karyn S. Kunzelman PhD ${ }^{\mathrm{b}}$
}

Objectives: Progressive aortic root dilatation and an increased aortic root elastic modulus have been documented in persons with Marfan syndrome. To examine the effect of aortic root dilatation and increased elastic modulus on leaflet stress, strain, and coaptation, we used a finite-element model.

Methods: The normal model incorporated the geometry, tissue thickness, and anisotropic elastic moduli of normal human roots and valves. Four Marfan models were evaluated, in which the diameter of the aortic root was dilated by $5 \%, 15 \%$, $30 \%$, and $50 \%$. Aortic root elastic modulus in the 4 Marfan models was doubled. Under diastolic pressure, regional stresses and strains were evaluated, and the percentage of leaflet coaptation was calculated.

Results: Root dilatation and stiffening significantly increased regional leaflet stress and strain compared with normal levels. Stress increases ranged from $80 \%$ to $360 \%$ and strain increases ranged from $60 \%$ to $200 \%$ in the 50\% dilated Marfan model. Leaflet stresses and strains were disproportionately high at the attachment edge and coaptation area. Leaflet coaptation was decreased by approximately $20 \%$ in the $50 \%$ root dilatation model.

Conclusions: Increasing root dilatation and root elastic modulus to simulate Marfan syndrome significantly increases leaflet stress and strain and reduces coaptation in an otherwise normal aortic valve. These alterations may influence the decision to use valve-sparing aortic root replacement procedures in patients with Marfan syndrome.

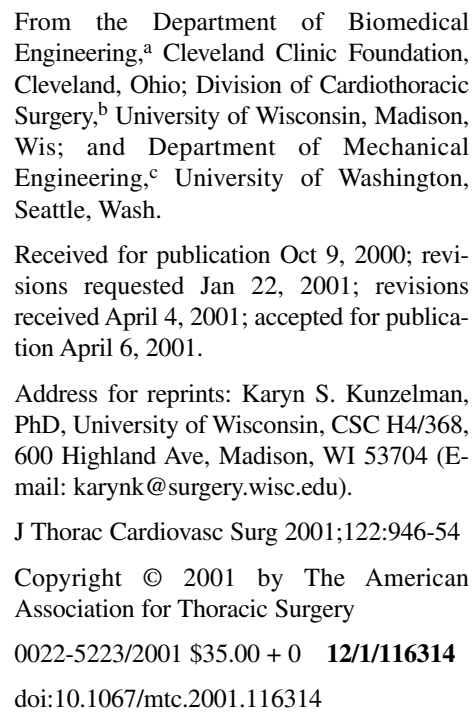

Received for publication Oct 9, 2000; revisions requested Jan 22, 2001; revisions received April 4, 2001; accepted for publication April 6, 2001

Address for reprints: Karyn S. Kunzelman, $\mathrm{PhD}$, University of Wisconsin, CSC H4/368, 600 Highland Ave, Madison, WI 53704 (Email: karynk@surgery.wisc.edu).

J Thorac Cardiovasc Surg 2001;122:946-54

Copyright (C) 2001 by The American Association for Thoracic Surgery

0022-5223/2001 \$35.00 + $0 \quad \mathbf{1 2 / 1 / 1 1 6 3 1 4}$

doi:10.1067/mtc.2001.116314

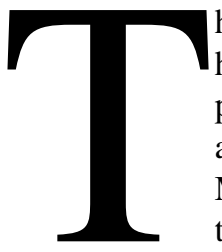

he numerous abnormalities associated with the Marfan syndrome $e^{1,2}$ have been linked to a genetic defect in fibrillin. ${ }^{3}$ Fibrillin is a component of the microfibrils making up the elastic meshwork in the aorta and other large vessels. ${ }^{4-6}$ The defect in fibrillin found in the Marfan syndrome results in disorganization and fragmentation of the elastic meshwork. Normally, elastic fibers are responsible for maintaining blood vessels at their normal dimensions by providing the ability to expand with the cyclic increase of blood pressure and then allowing complete recovery to the initial state on removal of the pressure load. In contrast, collagen fibers, which are much stiffer than elastic fibers, are responsible for preventing the aortic wall from stretching too far. ${ }^{7}$ Elastic fragmentation therefore reduces the ability of the aortic wall to fully recover from the cyclic distending pressure, resulting in permanent stretching and dilatation. This dilatation shifts the pressure load from the fragmented elastic network to the stiffer collagen fibers, resulting in an increased elastic modulus in the aortic wall. ${ }^{4,6,7}$

The altered aortic root geometry and elastic modulus may be linked to observed valvular changes, such as increased size, fibrotic thickening of the coaptation area, 
TABLE 1. Material properties and element thicknesses for the aortic valve and normal root $^{16-20}$

\begin{tabular}{lcc}
\hline Property & Aortic valve & Aortic root \\
\hline $\mathrm{E}_{\text {circ }}(\mathrm{kPa})$ & 6885 & 334 \\
$\mathrm{E}_{\text {rad }}(\mathrm{kPa})$ & 1624 & - \\
$\mathrm{E}_{\text {long }}(\mathrm{kPa})$ & - & 350 \\
$\mathrm{G}_{\mathrm{xy}}(\mathrm{kPa})$ & 1121 & 119 \\
$\mathrm{G}_{\mathrm{yz}}(\mathrm{kPa})$ & 1121 & 115 \\
$\mathrm{G}_{\mathrm{xz}}(\mathrm{kPa})$ & 560 & 119 \\
$v_{x y}$ & 0.106 & 0.450 \\
$v_{\mathrm{yz}}$ & 0.106 & 0.450 \\
$v_{x z}$ & 0.45 & 0.429 \\
Thickness $(\mathrm{mm})$ & $0.18-2.75$ & $0.60-2.14$ \\
\hline
\end{tabular}

In the Marfan models the aortic root elastic moduli were doubled $(100 \%$ increase) ${ }_{1}^{4}$ and the shear moduli and Poisson ratios were recalculated appropriately. $\mathrm{E}_{\text {circ }}$, Circumferential elastic modulus; $\mathrm{E}_{\mathrm{rad}}$, radial elastic modulus; $E_{l o n g}$ longitudinal elastic modulus; $\mathrm{G}_{\mathrm{xy}}, \mathrm{G}_{\mathrm{yz}}$, and $\mathrm{G}_{\mathrm{xz}}$ orthotopic shear moduli; $v_{x y}, v_{y z^{\prime}}$ and $v_{x z^{\prime}}$ orthotropic Poisson ratios.

and marginal rolling sometimes found in aortic valves in the Marfan syndrome. ${ }^{5,8,9}$ The normal function of the valve is dependent on the compliant tissue properties and rounded shape of the normal, undilated aortic root. ${ }^{10,11}$ In the case of the Marfan syndrome, the combined effect of aortic root dilatation and increased aortic root elastic modulus could increase the stress on the aortic valve leaflets, potentially resulting in leaflet alterations. Although the tissue constituent makeup, geometry, and material properties of the aortic root in Marfan syndrome have been characterized, there has not yet been a systematic study of the relationship between these pathologic changes and the biomechanical function of the aortic valve. The objective of this investigation was to examine the influence of dilatation of a stiffened Marfan syndrome aortic root on aortic valve function (stress, strain, and coaptation) by using a 3-dimensional and anatomically realistic finite-element model of the aortic root and valve. ${ }^{12}$

\section{Methods}

The detailed development of the original 3-dimensional model of the aortic root and valve has been described previously ${ }^{12}$ and is summarized here.

\section{Model Geometry and Element Development}

Finite-element analysis is a computational technique in which an object with a complicated structure is divided into smaller sections (ie, elements) that are interconnected by common points (ie, nodes). This discretization enables the use of algebraic equations to describe the individual structural state at each node. The solution of the system of equations yields the stress and strain at any point in the entire object. Our finite-element model was developed by means of ANSYS software (version 5.3; ANSYS Inc, Canonsburg, Pa) run on a DEC Alphastation 400 4/233 (Digital Equipment Corporation,
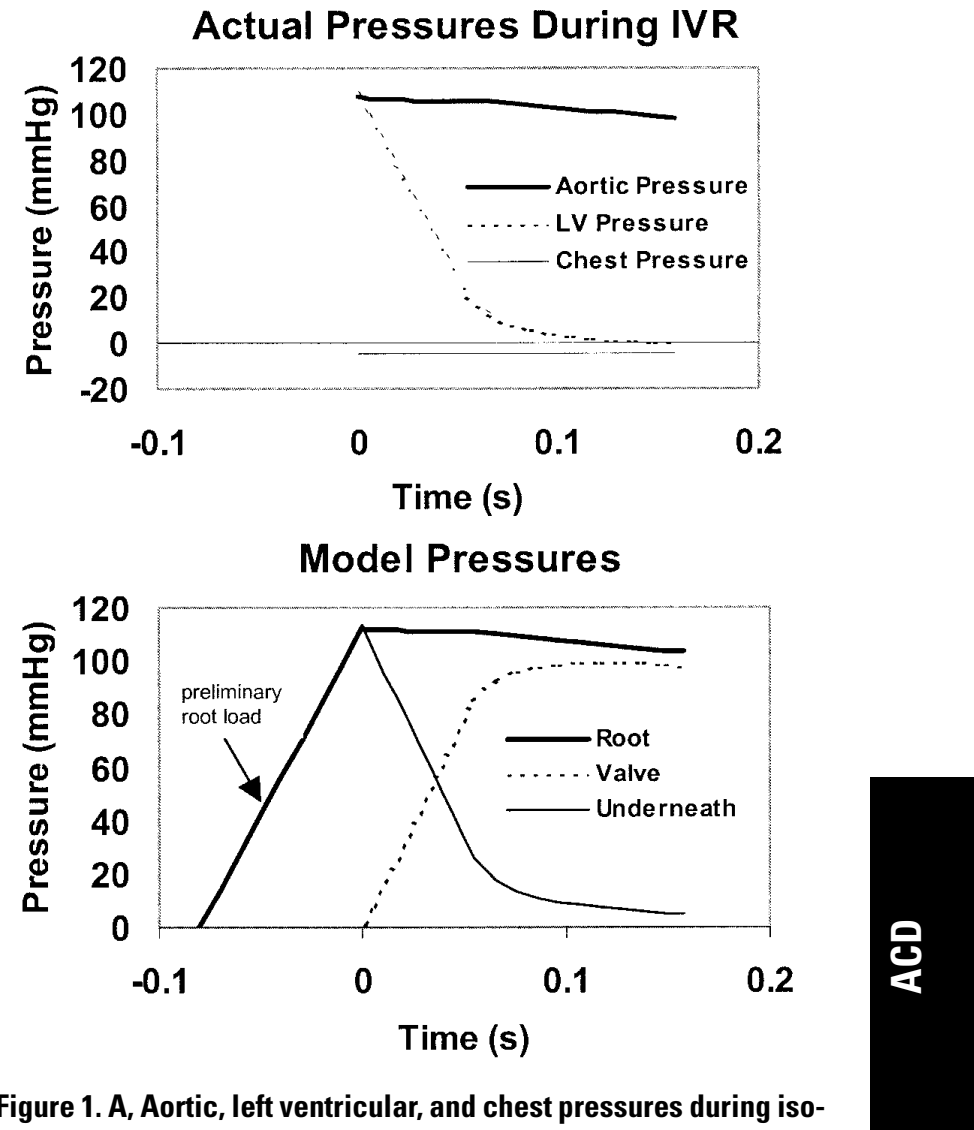

Figure 1. A, Aortic, left ventricular, and chest pressures during isovolumic relaxation (IVR) used to calculate the pressures applied to the model root and valve. Isovolumic relaxation is assumed here to begin at $\mathbf{O}$ seconds. $B$, Pressure-loading curves for the aortic root, aortic valve, and the region of the root underneath the valve (the ventricular-aortic septum). Loading was initiated with the linearly increasing root pressure and was followed by the addition of valve pressure at the onset of isovolumic relaxation at time $\mathbf{0}$. Model loading finished at the end of isovolumic relaxation, when the maximum transvalvular pressure was reached.

Maynard, Mass). Normal human aortic valve and root specimens in a zero-pressure state were used to establish the geometry for the model by using magnetic resonance imaging to obtain the data coordinates and hence the nodes to represent the shape of the aortic root and valve. Elastic 6-node triangular shell elements were generated from these nodes to create 5000 elements in the aortic root and 1815 elements in the 3 valve leaflets.

\section{Normal Model Boundary Conditions}

Geometric boundary constraints were assigned to all models. First, contact elements were incorporated into the leaflet surfaces to permit sliding and to prevent the leaflets from passing through each other or through the root wall. Second, the lowest layer of the aortic root base was restricted to permit only radial displacement. Finally, the physiologic longitudinal stretch found normally in $\operatorname{arteries}^{13}$ was imposed by applying tension at the top of the ascending aorta and at the distal ends of the coronary ostia. 


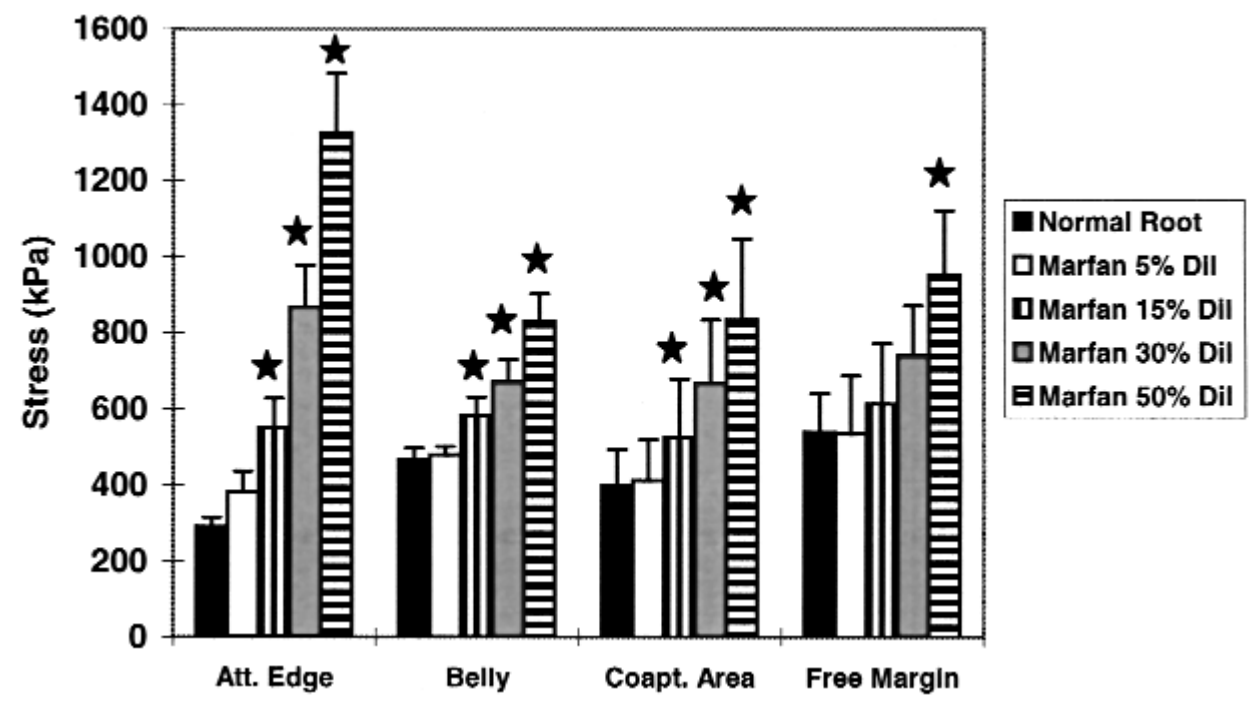

Figure 2. Aortic valve regional stresses in the normal and progressive Marfan syndrome models. *Significant difference compared with the normal root model.
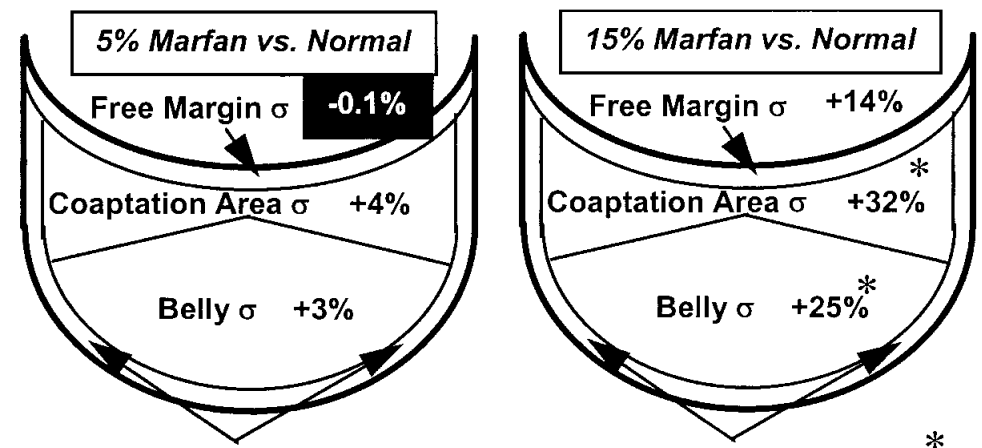

Attachment Edge $\sigma+32 \%$
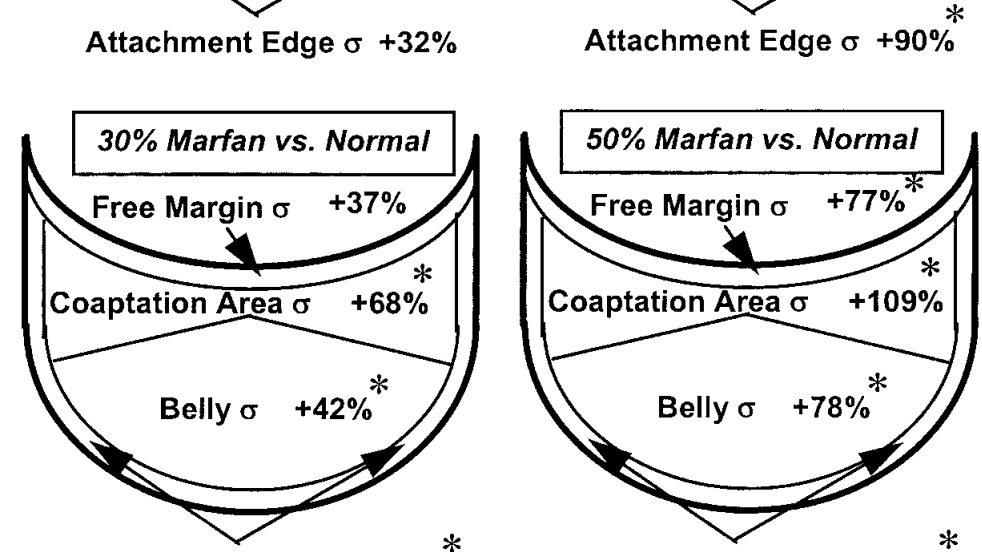

Attachment Edge $\sigma+199 \%$

Attachment Edge $\sigma+357 \%$

Figure 3. Schematics of altered leaflet stress patterns in the Marfan models compared with the normal root model.

\section{Marfan Model Boundary Conditions}

To simulate aortic root dilatations of $5 \%, 15 \%, 30 \%$, or $50 \%$, we applied radially directed forces to the root in the 4 Marfan models. ${ }^{14}$ This action also served to pull the leaflets apart and create the slight central deficiency found clinically. $8,9,15$ Only the root geometry was altered; all other conditions remained as in the normal model. Because these radial forces established preliminary stresses and strains that might not be present in an actual aortic root dilated as a result of Marfan syndrome, the force-induced root results were subtracted from the final results to obtain the root stresses and strains caused by the pressure load alone. However, because our objective was to determine the effect of the Marfan-associated root 


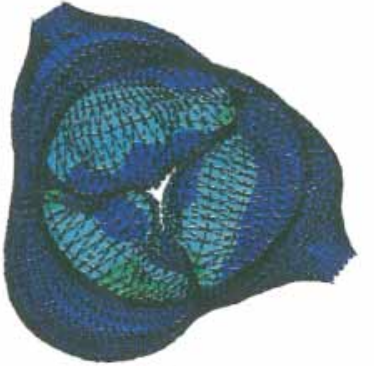

(a)

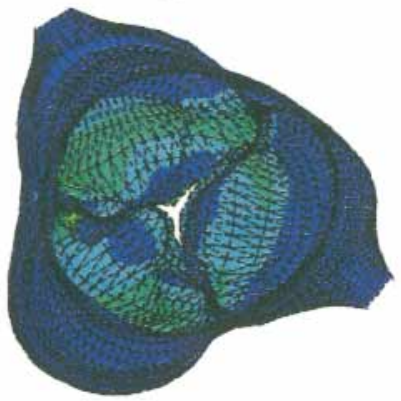

(c)

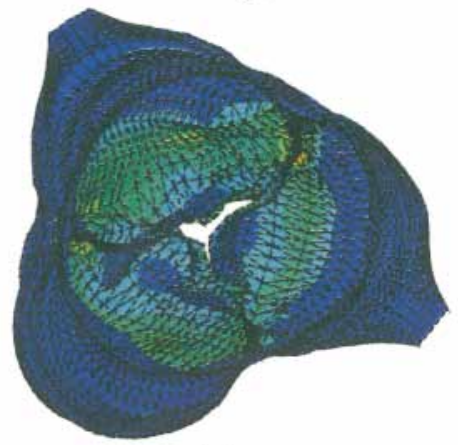

(e)

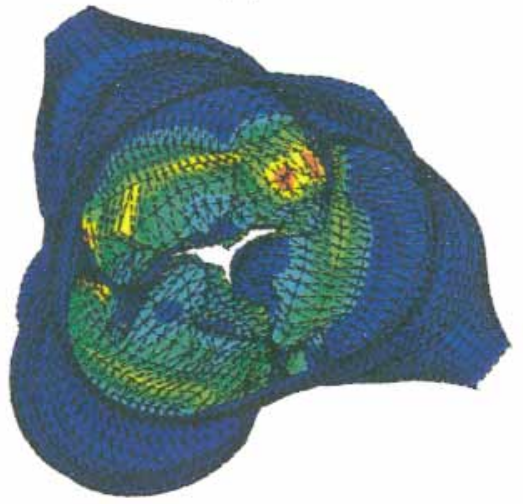

(g)
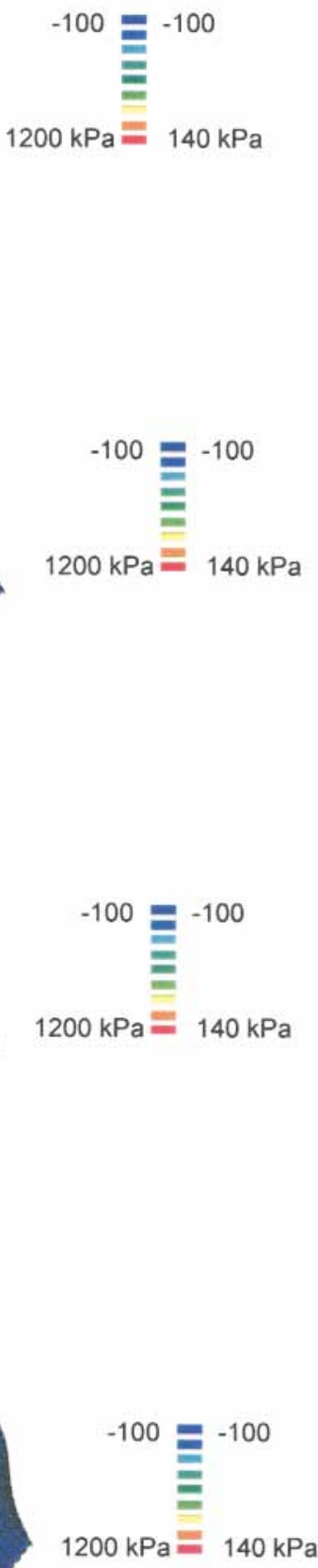

$1200 \mathrm{kPa}=140 \mathrm{kPa}$

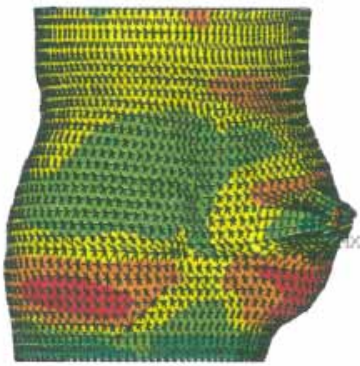

(b)

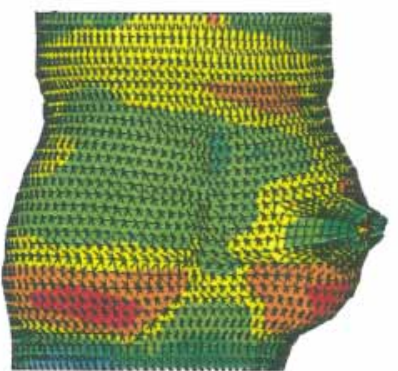

(d)

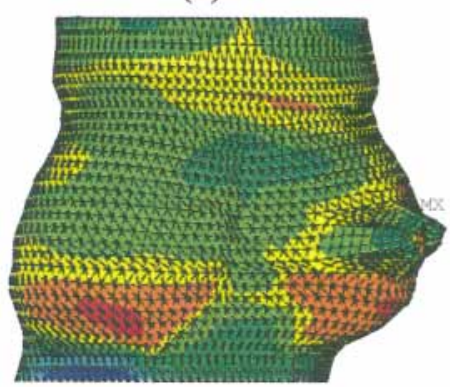

(f)

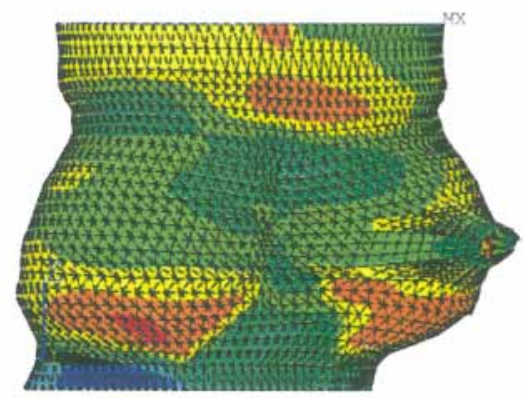

(h)

Figure 4. Stress contours for the aortic root and valve in the progressive Marfan syndrome models: (a) $\mathbf{5} \%$, top view; (b) $5 \%$, side view; (c) $15 \%$, top view; (d) $15 \%$, side view; (e) $30 \%$, top view; (f) $30 \%$, side view; (g) $50 \%$, top view; and (h) $50 \%$, side view. All models in this figure have the same scaling. 


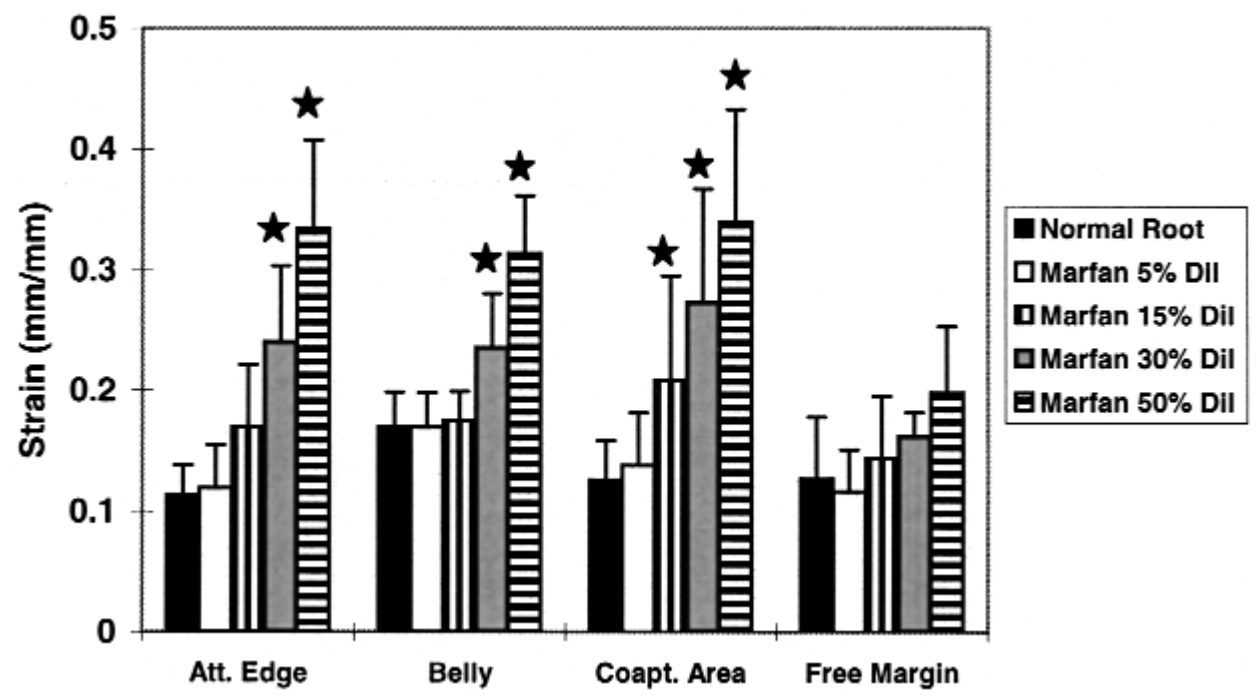

Figure 5. Aortic valve regional strains in the normal and progressive Marfan syndrome models. *Significant difference compared with the normal root model.

alterations on the leaflet stress and strain, the force-induced preliminary values were not subtracted from the leaflet results.

\section{Pressure Loading}

To represent the early diastolic loading of the aortic valve and root (when peak pressure across the valve is generated), we applied simulated physiologic pressures to the valve and root structure in 2 phases (Figure 1). In the first phase, the aortic root alone was pressurized by a linearly increasing load from the zero-pressure state to the end-systolic aortic pressure level. In the second phase, physiologic pressure loads were applied to the valve, to the end-systolic root, and to the region of the root underneath the valve. These physiologic pressures were calculated as the pressure differences between the aortic root and left ventricle (load applied to aortic valve), between the aortic root and chest (root), and between the left ventricle and chest (subvalvular root). Loading started at endsystole, just after valve closure, and finished at the end of isovolumic relaxation, when peak pressure across the valve was reached.

\section{Thickness and Material Properties}

The aortic root-thickness values were measured directly from the magnetic resonance images of the normal aortic root wall. The thicknesses of the unpressurized valve leaflets were determined from published data on the normal aortic valve. ${ }^{16}$ The material properties of the leaflet and root tissues were also calculated from published stress-strain data of normal tissues. ${ }^{17-19}$ Elastic moduli values were determined from linear fits to the physiologic range of the tissues' stress-strain curves in directions either parallel or perpendicular to the collagen fiber alignments, resulting in an anisotropic analysis (Table 1). ${ }^{16-20}$ A Poisson ratio of 0.45 was used to account for tissue incompressibility. ${ }^{20}$ In the Marfan models the aortic root elastic modulus was doubled (100\% increase) to simulate the shift toward collagen load bearing (caused by elastic fragmentation and subsequent root dilatation). ${ }^{4}$ The bending stiffness of the valve elements was reduced by $98.5 \%$ (method detailed previously) ${ }^{12}$ to represent the pliability of the aortic valve leaflets. ${ }^{21}$

\section{Solution Method}

The solution phase was performed on the DEC Alphastation or on the CRAY J-90 at the Texas Advanced Computing Center. The solution was performed iteratively by dividing the pressureloading phases into a number of equal steps (81 aortic root preliminary steps +118 physiologic root/valve steps $=199$ total $)$.

\section{Output Analysis}

For both the leaflets and root walls, the magnitude and location of the principal tensile stresses and strains were recorded at the end of physiologic loading, when maximum transvalvular pressure occurred. Therefore stresses and strains in the model at that time point are likely representative of the maximum values attained during the entire cardiac cycle. Stress $(\sigma)$ is defined as the force $(F)$ per unit area $(\mathrm{A})$ :

$$
\sigma=\mathrm{F} / \mathrm{A}
$$

Strain $(\varepsilon)$ is defined as the percentage extension or stretch of the tissue:

$$
\varepsilon=\Delta \mathrm{L} / \mathrm{Lo}
$$

where $\Delta \mathrm{L}$ is change in length, and Lo is original length. Elastic modulus $(\mathrm{E})$ is defined as the change in stress $(\Delta \sigma)$ divided by the change in strain $(\Delta \varepsilon)$ :

$$
\mathrm{E}=\Delta \sigma / \Delta \varepsilon
$$

Regional magnitudes were calculated by grouping sets of elements to define specific model components (belly, coaptation area, free margin, attachment edge, annular sinus wall, and sinotubular junction sinus wall). These components were analyzed to calculate the peak average, which was the average value of the $5 \%$ of elements 


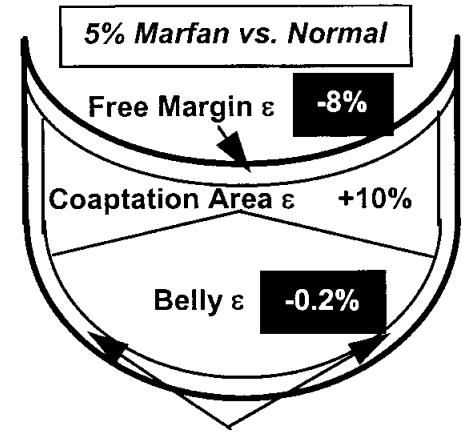

Attachment Edge $\varepsilon \quad+5 \%$

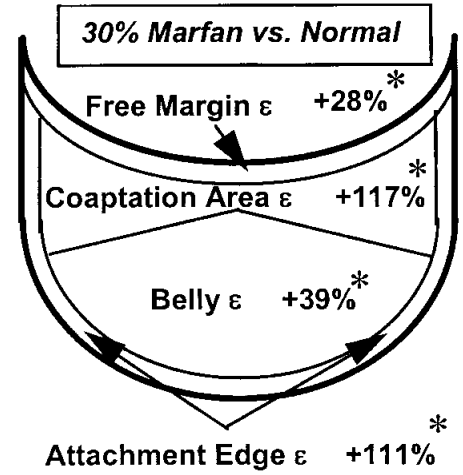

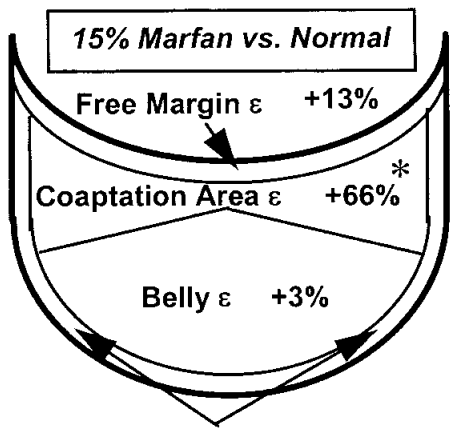

Attachment Edge $\varepsilon \quad+49 \%$

Figure 6. Schematics of altered leaflet strain patterns in the Marfan models compared with the normal root model.

with the highest values (peak standard deviation was calculated similarly). The coaptation area, defined as the percentage of leaflet area contacting the adjacent leaflet surfaces, was also examined throughout the preliminary root-loading and subsequent physiologic root/valve-loading phases. Leaflet coaptation creates a large, tight seal across the closed valve; reduced coaptation could indicate the potential for valvular regurgitation. The stress and strain results were compared with analysis of variance to determine significant differences with respect to root dilatation and aortic root elastic modulus.

\section{Results}

\section{Valve Stress, Strain, and Coaptation}

The models simulating Marfan syndrome demonstrated progressive increases in peak average valve stress, corresponding to increasing amounts of root dilatation compared with that seen in the normal root model (Figures 2-4). In the 5\% dilatation model the increase in leaflet stress was nominal, on the order of $0 \%$ to $32 \%$. However, by the $50 \%$ dilatation model, the increase in valve stresses was much higher, ranging from $77 \%$ to $357 \%$. The greatest increases in leaflet stress in all the Marfan models were evident at the attachment edge. This pattern was different from that found in the normal root and valve, ${ }^{12}$ where leaflet stresses were lower at the attachment edge, and were somewhat lower at the coap- tation area than in the leaflet belly and free margin caused by the support of the adjacent leaflet. In contrast, the Marfan syndrome models showed significant increases in stress at the coaptation area, as a result of reduced coaptation.

The Marfan models also demonstrated progressive increases in valve strain, with increasing root dilatation, compared with that seen in the normal model (Figures 5 and 6). With limited dilatation (5\% model), there were actually slight decreases in strain in some leaflet areas and only a nominal increase in strain in other areas, on the order of 5\% to $10 \%$. However, with greater dilatation (50\% model), the increase in strain was much greater, ranging from $57 \%$ to $195 \%$. The greatest increases in strain were seen at the attachment edge and coaptation area of the leaflets in each Marfan model.

Changes in leaflet coaptation accompanied the alterations in leaflet stress and strain. In the Marfan models with 5\% and $15 \%$ root dilatation, leaflet coaptation was not dramatically affected $(<2 \%$ change compared with normal values). However, progressive dilatation resulted in significantly reduced leaflet coaptation. When the root was dilated by $30 \%$, leaflet coaptation decreased by $8 \%$, and in the $50 \%$ root dilatation model, leaflet coaptation was decreased by $17 \%$, compared with normal values. This reduced coaptation could 
Root Sinus Stresses

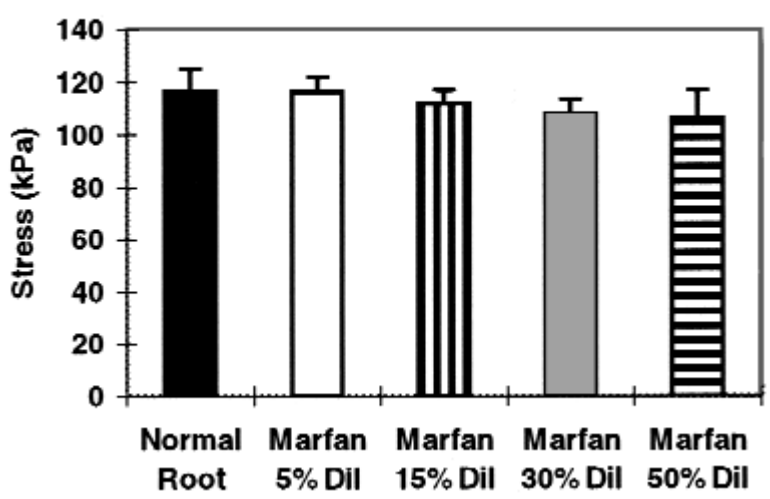

Root Sinus Strains

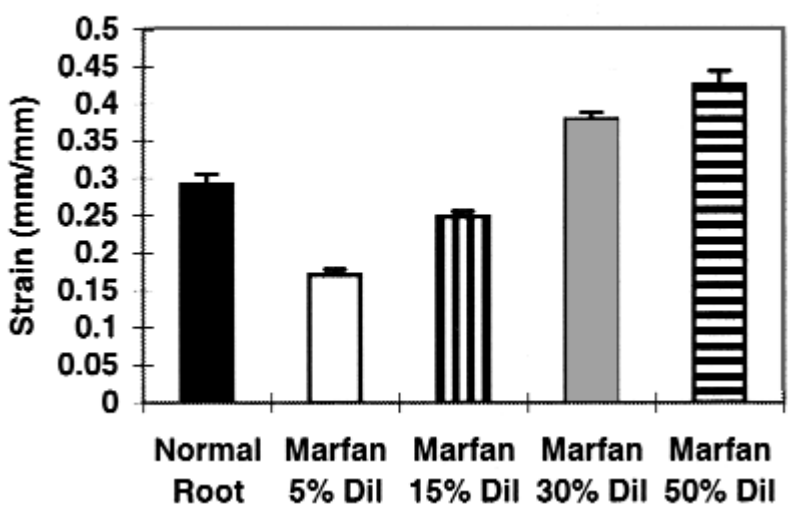

Figure 7. Aortic root stress $(A)$ and strain $(B)$ in the normal and progressive Marfan syndrome models. *Significant difference compared with the normal root model.

be seen as a tendency toward central valve opening, with increasing dilatation of the Marfan aortic root (Figure 4).

\section{Root Stress and Strain}

The stress values calculated for the aortic root walls (caused by pressure loading only) varied by less than $10 \%$ between the Marfan models and the normal root model (Figure 7). Strain magnitudes, however, did differ between models. In the $5 \%$ and $15 \%$ Marfan models the strain in the root was decreased by $36 \%$ and $15 \%$, respectively, compared with that of the normal root. In contrast, for the $30 \%$ and $50 \%$ Marfan models, the strain in the root was increased by $30 \%$ and $45 \%$, respectively, compared with that of the normal root.

\section{Discussion}

These finite-element models demonstrated that the increased aortic root elastic modulus and progressive root dilatation found in Marfan syndrome results in an increase in stress and strain in aortic valve leaflets, as well as significantly reduced leaflet coaptation.

The initial root dilatation in the Marfan models (5\% and $15 \%$ ) was accompanied by progressively increasing leaflet stresses, particularly at the attachment edge and belly. The overall leaflet stress pattern in these 2 Marfan models, however, was similar to that of the normal valve leaflets in that the stresses at the attachment edge of the leaflet were lower in magnitude than the rest of the leaflet tissue (caused by stress sharing between the root and valve). In contrast, further dilatation of the Marfan root (30\% and 50\%) greatly increased the leaflet stresses, making them disproportionately high at the attachment edge and coaptation area. These regionally high magnitudes are due to reductions both in stress sharing at the attachment edge and in mutual compressive stress relief in the coaptation area. The increased leaflet stresses at the attachment edge were accompanied by a slight decrease in aortic root stress, which indicates a transfer of root stress to the valve leaflet, the opposite of the situation in the normal model.

In addition to increased leaflet stresses, the strains in the leaflets were altered, which directly affected leaflet coaptation. In the 5\% dilated Marfan model strains were increased at the attachment edge and coaptation area but were actually decreased at the belly and free margin. These mixed results suggest that for small geometric changes to the root, a compensatory mechanism may occur through which valve deformation can be slightly adapted to respond to an altered environment. It may be through such a mechanism that the regional leaflet strains were altered to maintain coaptation, albeit at the expense of increased leaflet stress. However, with further increases in diameter of the Marfan root, the adaptation mechanism was not sufficient to counteract the dilatation, and the strain and coaptation patterns were significantly affected. With $15 \%$ and greater dilatation, strains were increased across the entire leaflet. By $30 \%$ and $50 \%$ dilatation, even though the leaflet was tightly stretched, not enough tissue was present to maintain normal coaptation, suggesting that the proposed compensatory mechanism for preservation of coaptation failed. Because the valves in these models could no longer maintain normal coaptation, these results indicate the possibility for regurgitation in these moderately dilated Marfan aortic roots.

The amount of strain in the aortic root sinuses also contributed to the results in this series of models. In the $5 \%$ and $15 \%$ dilated Marfan models aortic root strain was slightly decreased, which would suggest that the root was compensating to preserve coaptation. In contrast, increased root strains in the more dilated Marfan models (30\% and 50\%) again suggests that the compensatory mechanism has failed at these larger root diameters, and the valve cannot maintain normal coaptation. 
The stress and strain results in the Marfan models were also compared with those of a series of idiopathic dilatation models that we previously reported. ${ }^{14}$ Overall, the dilatation component of the Marfan models caused far greater increases in leaflet stresses than did the material change (30\%-60\% increases between subsequent dilatations vs 4\%-12\% differences between Marfan models and idiopathic dilatation models). However, the leaflet attachment edge stresses and strains were greater in the Marfan models than in corresponding idiopathic dilatation models. In particular, these stresses were significantly greater in the $5 \%$ and $15 \%$ Marfan models. The differences between the results of these 2 series of models emphasizes the contributing role of the elastic modulus of the aortic root in governing the stresses at the leaflet attachment edge. Alterations to the root modulus appear to be especially important in the early stages of aortic root dilatation and would undoubtedly influence leaflet stresses in the absence of root dilatation.

\section{Limitations}

As with any modeling study, there are some inherent limitations in our model, as described in our initial report. ${ }^{12}$ First, we chose to simulate only the final closing phase of the valve because the transvalvular pressure and tensile stresses are highest during this period. Second, because of software restrictions, the material properties were assumed to be constant in the physiologic range of the stress-strain curve. As a result, the modeled stresses and strains were likely lower than those in the actual root and valve. However, these models analyzed diastolic function only, in which the valve tissue reportedly functions in the linear and higher elastic modulus region of its stress-strain curve. ${ }^{17}$ Furthermore, the normal aortic root elastic modulus was calculated from reported in vivo measurements. ${ }^{18}$ Third, the analysis assumed that the initial zero-pressure state in the root and valve was equivalent to a zero-stress state. Because of residual stresses caused by the differential makeup of the tissue layers $^{21,22}$ and normal dynamic root-valve motion, a zerostress state may not exist in vivo. However, the magnitude of these initial stresses was assumed to be negligible in comparison with the tissue stresses at peak pressure. Fourth, a small deformation analysis was used on the basis of the rationale that the applied simulated pressures did not lead to significant changes in the model geometry. We therefore assumed that any incrementally increased deformations that could be gained from a large deformation analysis would not be large enough to justify the extracomputational burden. Finally, mild root dilatation in Marfan syndrome is believed to be limited to the sinus region and only later spread upward to the proximal ascending aorta. ${ }^{6,9}$ However, the degree of root dilatation in all the Marfan models was assumed to be uniform from the anulus to above the sinotubular junction (the root base was not dilated). This action likely overestimated the stresses and strains above the upper root in the $5 \%$ and $15 \%$ dilated Marfan models.

Despite these limitations, the normal and Marfan models were designed by using identical conditions; only specific geometric and material properties (aortic root diameter and elastic modulus) were altered between models. Therefore, comparison between models provides a relative estimate of the differences that could be expected in vivo as a result of dilatation of a Marfan aortic root.

\section{Clinical and Surgical Implications}

These finite-element model results indicate that even with limited root dilatation associated with the Marfan syndrome, the simulated leaflets experience stress and strain changes before any loss of coaptation. These changes have been associated with leaflet calcification, ${ }^{23}$ may lead to tissue remodeling to bear the higher stresses, and could explain the reports of aortic valve thickening $5,8,9$ and regurgitation 1,24,25 found in persons with the Marfan syndrome. The greater attachment edge stresses and strains in the Marfan models compared with those in the idiopathic dilatation models suggest that there may be an earlier critical stage for the valve-sparing root-replacement procedure in persons with the Marfan syndrome. However, in patients with Marfan syndrome with advancing root dilatation, their aortic valve leaflets may no longer be normal and might not benefit as much as desired from a valve-sparing procedure. Of course, an additional factor in determining the optimal surgical timing is the inherent risk and complications of the surgical procedure. Further research regarding the timing of tissue remodeling in the aortic valve would be helpful to surgeons in determining the optimal time and procedure for replacement of the diseased aortic root in Marfan syndrome.

We thank the Whitaker Foundation and the National Partnership for Advanced Computational Infrastructure for their support of this research. This research was performed in the Department of Bioengineering and the Division of Cardiothoracic Surgery at the University of Washington.

\section{References}

1. Pyeritz R, McKusick V. The Marfan syndrome: diagnosis and management. N Engl J Med. 1979;300:772-7.

2. Godfrey M. The Marfan syndrome. In: Beighton P, editor. McKusick's heritable disorders of connective tissue. 5th ed. St Louis: Mosby; 1993. p. 51-136.

3. Dietz HC, Cutting CR, Pyeritz RE, et al. Marfan syndrome cuased by a recurrent de novo missense mutation in the fibrillin gene. Nature. 1991;352:337-9.

4. Sonesson B, Hansen F, Lanne T. Abnormal mechanical properties of the aorta in Marfan's syndrome. Eur J Vasc Surg. 1994;8:595-601.

5. Wagenvoort C, Neufeld H, Edwards J. Cardiovascular system in Marfan's syndrome and in idiopathic dilatation of the ascending aorta. Am J Cardiol. 1962;9:496-506.

6. Hwa J, Richards JG, Huang $\mathrm{H}$, et al. The natural history of aortic dilatation in Marfan syndrome. Med J Aust. 1993;158:558-62.

7. Dobrin PB. Pathophysiology and pathogenesis of aortic aneurysms. Current concepts. Surg Clin North Am. 1989;69:687-703. 
8. Yacoub MH, Sundt TM, Rasmi N. Management of aortic valve incompetence in patients with Marfan syndrome. In: Hetzer R, Gehle P, Ennker J, editors. Cardiovascular aspects of Marfan syndrome. Darmstadt: Steinkopff; 1995. p. 71-81.

9. Roberts WC, Honig HS. The spectrum of cardiovascular disease in the Marfan syndrome: a clinico-morphologic study of 18 necropsy patients and comparison to 151 previously reported necropsy patients. Am Heart J. 1982;104:115-35.

10. Thubrikar MJ, Nolan SP, Aouad J, Deck JD. Stress sharing between the sinus and leaflets of canine aortic valve. Ann Thorac Surg. 1986;42:434-40.

11. Bellhouse BJ. The fluid mechanics of the aortic valve. In: Ionescu ML, Ross DN, Woller GH, editors. Biological tissue in heart valve replacement. London: Butterworth-Heinemann; 1972. p. 23-47.

12. Grande KJ, Cochran RP, Reinhall PG, Kunzelman KS. Stress variations in the human aortic root and valve: the role of anatomic symmetry. Ann Biomed Eng. 1998;26:534-45.

13. Han HC, Fung YC. Longitudinal strain of canine and porcine aortas. $J$ Biomech. 1995;28:637-41.

14. Grande-Allen K, Cochran R, Reinhall P, Kunzelman K. Mechanisms of aortic valve incompetence: finite element modeling of aortic root dilatation. Ann Thorac Surg. 2000;69:1851-7.

15. Legget ME, Unger TA, O'Sullivan CO, et al. Aortic root complications in Marfan's syndrome: identification of a lower risk group. Heart. 1995;75:389-95
16. Clark RE, Finke EH. Scanning and light microscopy of human aortic leaflets in stressed and relaxed states. J Thorac Cardiovasc Surg. 1974;67:792-804

17. Dobrin PB. Vascular mechanics. In: Shepherd JT, Abboud F, editors. Handbook of physiology. Vol. 3. Washington, DC: American Physiological Society; 1983. p. 65-102.

18. Kalath S, Tsipouras P, Silver FH. Non-invasive assessment of aortic mechanical properties. Ann Biomed Eng. 1986;14:513-24.

19. Vesely I, Noseworthy R. Micromechanics of the fibrosa and the ventricularis in aortic valve leaflets. J Biomech. 1992;25:101-13.

20. Vawter DL. Poisson's ratio and incompressibility. J Biomech Eng. 1983;105:194-5.

21. Vesely I, Boughner D. Analysis of the bending behaviour of porcine xenograft leaflets and of neutral aortic valve material: bending stiffness, neutral axis and shear measurements. J Biomech. 1989;22:655-71.

22. Fung YC. In: Biomechanics: mechanical properties of living tissues. 2nd ed. New York: Springer-Verlag; 1993.

23. Sabbah HN, Hamid MS, Stein PD. Mechanical stresses on closed cusps of porcine bioprosthetic valves: correlation with sites of calcification. Ann Thorac Surg. 1986;42:93-6.

24. Gott V, Laschinger J, Cameron D, et al. The Marfan syndrome and the cardiovascular surgeon. Eur J Cardiothorac Surg. 1996;10:149-58.

25. Becker A. Cardiovascular pathology in Marfan syndrome-an overview. In: Hetzer R, Gehle P, Ennker J, editors. Cardiovascular aspects of Marfan syndrome. Darmstadt: Steinkopff; 1995. p. 25-31.

\section{Targeted}

The Journal of Thoracic and Cardiovascular Surgery gives you two tables of contents.

The condensed table of contents tells you at a glance what topics and authors are presented each month. The expanded table of contents gives you a brief abstract of each article. You select only those articles of most interest to you for further reading. 